\title{
INFLUENCE OF HONEY-ROASTING ON THE MAIN PHARMACOLOGICAL ACTIVITIES AND THE WATER-SOLUBLE ACTIVE GLYCOSIDES OF LICORICE
}

\author{
Mengyue Wang, Min Zhang, Qiyu Tang and Xiaobo $\mathrm{Li}^{*}$
}

\author{
School of Pharmacy, Shanghai Jiao Tong University, Shanghai 200240, P. R. China \\ *E-mail: $\underline{x b l i @ \text { situ.edu.cn }}$
}

\begin{abstract}
In traditional Chinese medicine (TCM), licorice is usually processed with honey and traditionally used in decoction form. However, the influence of honey-roasting on the main pharmacological activities and the water-soluble active constituents of licorice has not been reported. The aim of the present study is to determine whether honey-roasting can modify the main pharmacological activities and the active constituents of licorice. According to licorice clinical application and processing method, the mainly related pharmacological activities of crude licorice, processed licorice and refined honey, such as enhancing immune function, relieving cough, eliminating phlegm and detoxication, were compared. The results showed that honey-roasting obviously reinforced the licorice activity of enhancing Pi-deficiency mice's immune function, and significantly weaken the licorice activity of relieving cough, removing phlegm and detoxication. However, honey didn't show the significant activity of relieving cough, removing phlegm and detoxication. The influence of honey-roasting on the chemical compositions in licorice slice and licorice decoction was investigated by using HPLC. The results showed that the content and the decocting quantity of mainly 5 active glycosides in licorice, i.e. liquiritin apioside, liquiritin, licuraside, isoliquiritin and glycyrrhizin, obviously changed after processing; glycyrrhizin and liquiritin obviously decomposed during honey-roasting. In conclusion, honey-roasting obviously modified the main pharmacological activities and the water-soluble compositions of licorice. The modification was not cause by honey only. This finding may shed some light on understanding the differences in the therapeutic values of crude and processed licorice.
\end{abstract}

Key words: crude licorice, processed licorice, honey-roasting, pharmacological activity, active glycosides, modification.

\section{Introduction}

Licorice, a very famous herbal medicine, has been used for more than 2 millennia in China and referred as the "King of Herb" (Shang et al., 2010). Licorice is usually administered as decoction, and commonly classified into two decoction slices, i.e. processed licorice (roasting with refined honey), crude licorice (China Pharmacopoeia Committee, 2005). According to TCM theory, the efficacies of crude licorice and processed licorice are very different. Crude licorice is good at relieving cough, dissipating phlegm and detoxication, and usually used to curing bronchitis, sore throat and intoxication caused by drugs and food, while processed licorice is expert in invigorating vital energy, and usually used to treat immune deficit, dyspepsia and arrhythmia (Gong, 2007).

Modern research revealed that the roasted licorice (roasted without honey) had the stronger effect in antianaphylaxis (Majima et al., 2004), anti-inflammation (Kim et al., 2006; Kim et al., 2010), neuroprotection (Hwang et al., 2006) and anti-diabetic action (Ko et al., 2007) than crude licorice. In recently years, pharmacological research also showed that processed licorice had the better effect of anti-arrhythmia (Huang et al., 1984) and analgesia (Peng et al., 1989) than crude licorice. However, the research about the influence of roasting with honey on the main efficacy of licorice, such as strengthening immunity, relieving cough, dissipating phlegm and detoxification, and the processing influence on the water-soluble active constituents of licorice, has not been reported. In the present study, therefore, the mainly pharmaceutical evaluation and the chemical analysis of licorice before and after honey-roasting were carried out. The results showed that honey-roasting could obviously modify the main pharmacological activities and chemical compositions of licorice.

\section{Materials and Methods \\ Animals}

Kunming albino mice (clean grade), with an average body mass of $20.0 \pm 2.0 \mathrm{~g}$ at the time of study initiation, provided by the Experiment Animal Center of Fudan University (Shanghai, Certificated number: SCXK (Hu) 2007-0026). Animals were kept under standard environmental conditions with free access to rodent diet and water. The animal experimental protocol was approved by the Institutional Animal Care and Use Committee of School of Pharmacy, Shanghai Jiao Tong University.

\section{Materials and agents}

Licorice was purchased from Inner Mongolia Yili Technology Company (Batch Number: GY 07020510) and identified as the root of Glycyrrhiza uralensis Fisch by one of authors-Prof. Xiaobo Li. The voucher specimen (SJTU 
2007-09-18) was deposited in School of Pharmacy, Shanghai Jiao Tong University. Refined honey was provided by Shanghai Jing'an Drug Manufactory. Reference standards, liquiritin apioside, liquiritin, licuraside, isoliquiritin, glycyrrhizin, liquiritigenin, glycyrrhetic acid-3-O- $\beta$-D-glucuronide and glycyrrhetic acid were isolated from licorice by the authors (Figure 1). The relative purities were all more than $95 \%$ by HPLC analysis. Indian ink was provided by Shanghai Maisha Biotechnology Company and diluted to 5 times before use. Sodium bicarbonate, ammonia water, phenol red, chloral hydrate, methanol, methanoic acid and phosphoric acid were analytical grade from Shanghai National Pharmaceutical Co. Ltd. Acetonitrile was HPLC grade from Shanghai Xingke Biochemistry Co., Ltd (Shanghai, China). Water was purified by Milli-Q system (Massachusetts, USA).

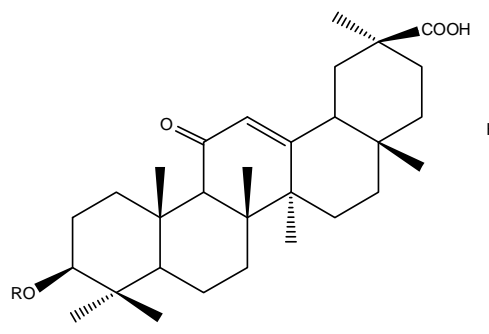

glycyrrhetic acid glycyrrhizin $\begin{array}{lll}\mathrm{R}=\mathrm{GluA} & \text { liquiritigenin } & \mathrm{R}=\mathrm{H} \\ \text { liquiritin } & \mathrm{R}=\mathrm{Glu}\end{array}$

$\mathrm{R}=$ GluA (2-1)-GluA liquiritin apioside $\mathrm{R}=\mathrm{Glu}-(2-1)$-Api

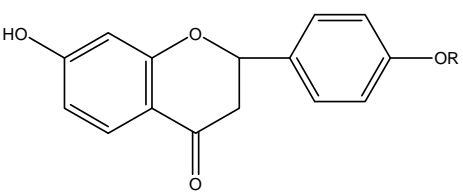

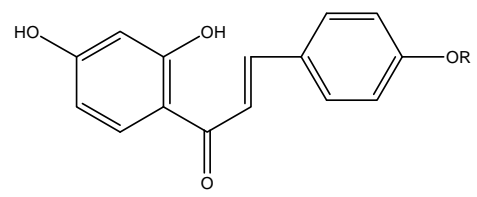

isoliquiritin licuraside
$\mathrm{R}=\mathrm{Glu}$ $\mathrm{R}=$ Glu-(2-1)-Ap

Figure 1: Chemical structures of 8 compounds

\section{Instruments}

Agilent 1200 series HPLC system consisted of quaternary pump, on-line degasser, autosampler, thermostatic column compartment and DAD (Agilent, Germany), 754 UV-VIS spectrophotometer (Shanghai Precision Scientific Instrument Corporation, China), BS300S analytic balance (Sartorius, Germany), transferpetto (Eppendorf, Germany), 402 ultrasonic nebulizer (Shanghai Heli Medical Apparatus and Instruments Factory, China), DZF-6050 vacuum drying oven (Shanghai Yiheng Scientific Instrument Corporation, China).

\section{Sample preparation}

Licorice processing: Each $100 \mathrm{~g}$ of crude licorice slice was macerated with $25 \mathrm{~g}$ refined honey in $50 \mathrm{ml}$ water for $8 \mathrm{~h}$, then roasted at $180{ }^{\circ} \mathrm{C}$ in drying oven for $1 \mathrm{~h}$ (China Pharmacopoeia Committee, 2005).

Sample for pharmacological test: $500 \mathrm{~g}$ crude and processed licorice were decocted 2 times in $5000 \mathrm{ml}$ of distilled water for $30 \mathrm{~min}$, respectively. The decoctions were filtered and condensed to $1.0 \mathrm{~g} / \mathrm{ml}$. The honey was diluted with distilled water to $1.0 \mathrm{~g} / \mathrm{ml}$.

Sample for chemical analysis of licorice decoction: $1.0 \mathrm{ml}$ of the decoction mentioned above was diluted to $25 \mathrm{ml}$ with distilled water, filtrated through a $0.22 \mu \mathrm{m}$ filteration and ready for the HPLC analysis (Liu et al., 2008).

Sample for chemical analysis of licorice slice: $0.5 \mathrm{~g}$ licorice powder was mixed with $25 \mathrm{ml}$ of $60 \%(\mathrm{v} / \mathrm{v})$ methanol aqueous solution in a conical flask with plunger, and subject to ultrasonic extraction for $30 \mathrm{~min}$. The sample solutions were obtained through a $0.22 \mu \mathrm{m}$ filteration and ready for the HPLC analysis (Liu et al., 2008).

Sample for stability analysis of active glycosides: $1.0 \mathrm{mg}$ of liquiritin and glycyrrhizin in powder form were mixed with $5.0 \mathrm{mg}$ honey, and heated at $180{ }^{\circ} \mathrm{C}$ in drying oven for $1 \mathrm{~h}$, respectively. The mixture was dissolved in $2 \mathrm{ml}$ of $50 \%$ methanol solution and filtrated for the HPLC analysis.

\section{Assay for the influence on immune function of Pi-deficiency mice}

One hundred and ten mice, including 55 males and 55 females, were randomly divided into 11 groups. Mice in group 1 (normal group) were administrated intragastrically (i.g.) with distilled water. Mice in group 2-11 were administrated (i.g.) with rhubarb extract (10 g/kg body weight, twice a day) for $5 \mathrm{~d}$ to induce Pi-deficiency model (Zheng et al., 2006). Then, the mice in group 1 and group 2 (model group) received (i.g.) distilled water only. The remaining mice received (i.g.) crude licorice, processed licorice and honey once daily for $7 \mathrm{~d}$, respectively. Twenty four hrs after the final administration, the mice were mainlined with the prepared India ink $(0.01 \mathrm{ml} / \mathrm{g}$ body weight $)$ in the cauda. Twenty microliter blood was taken from the eyepit vein plexus at $2 \mathrm{~min}$ and $20 \mathrm{~min}$ after the India ink injection, respectively. And then, the mice serum $\mathrm{OD}$ values at the wavelength of $680 \mathrm{~nm}$ were determined. The charcoal particle expurgation index $(\mathrm{K})$ of macrophage count was done according to the following formula: $\mathrm{K}=(\lg \mathrm{OD} 1-\lg \mathrm{OD} 2) /(\mathrm{t} 2-\mathrm{t} 1)$. Here $\mathrm{t} 1$ and $\mathrm{t} 2$ were the time points of taking blood from mouse eyes after injecting Indian ink ( $2 \mathrm{~min}$ and $20 \mathrm{~min}$, respectively). Thirty minutes after the India ink injection, the thymus gland and spleen were taken out and weighed. The thymus gland index and spleen index was calculated according to the following formula: Organ Index (mg/g) = Organ Weight $(\mathrm{mg}) /$ bodyweight $(\mathrm{g})$ (Chen, 1994).

\section{Antitussive test}

One hundred mice, including 50 males and 50 females, were divided into ten groups randomly. The mice in blank control group received (i.g.) distilled water, the remaining mice received (i.g.) crude licorice, processed licorice and honey once daily for $3 \mathrm{~d}$, respectively. One hr after the final administration, the antitussive activity in vivo was investigated on a 
classical mouse cough model induced by ammonia liquor. Briefly, mice were placed in a special glass chamber and exposed for 5 seconds to a $28 \%$ ammonia water aerosol which was produced through a nebulizer by compressed air at a pressure of about $400 \mathrm{mmHg}$. The cough incubation period and cough frequency produced during a 5 min exposure period were counted as reported by $\mathrm{Xu}$ et al. (2002).

\section{Eliminating phlegm test}

One hundred mice, including 50 males and 50 females, were divided into ten groups randomly. The mice in blank control group received (i.g.) distilled water, the remaining mice received (i.g.) crude licorice, processed licorice and honey once daily for $5 \mathrm{~d}$, respectively. Half an hour after the final administration, the mice were administrated $0.5 \%$ red phenol $(0.025 \mathrm{ml} / \mathrm{g}$ body weight) by intraperitoneal injection. Half an hour later, the trachea was separated and washed with $0.5 \mathrm{ml}$ of $0.5 \% \mathrm{NaHCO}_{3}$ solution for two times. The rinsed solution was incorporated and centrifuged at $4000 \times \mathrm{g}$ for $30 \mathrm{~min}\left(4^{\circ} \mathrm{C}\right)$. The OD value of the supernatant was determined at the wavelength of $545 \mathrm{~nm}$ (Xu et al., 2002).

\section{Detoxication test}

Eighty mice, including 40 males and 40 females, were divided into 4 groups randomly. The blank control group of animals received (i.g.) distilled water, the remaining groups received (i.g.) crude licorice, processed licorice and honey, respectively. Five minutes later, they were administrated (i.g.) chloral hydrate (1200 mg/kg body weight). Mortality was recorded in $2 \mathrm{~h}$ (Sanders et al., 1982).

\section{HPLC analysis}

The chemical composition profiles of licorice slice, the contents of the 5 active compounds in licorice slice and the decocting quantity in water decoction were determined by HPLC analysis. The sample was separated on an Agilent Zorbax Elipse XDB- $\mathrm{C}_{18}$ column $(5 \mu \mathrm{m}, 250 \times 4.6 \mathrm{~mm})$ connected with an Alltech Brava BDS- $\mathrm{C}_{18}$ guard column $(5 \mu \mathrm{m}, 7.5 \times$ $4.6 \mathrm{~mm}$ ). Then, the contents of 5 active compounds in the licorice slice and their decocting quantity in water decoction were calculated using their standards.

\section{Statistical analysis}

The results were expressed as mean \pm standard deviation (SD). Differences between groups were evaluated by one-way analysis of variance (ANOVA) followed by Dunnett's test and $\chi^{2}$ test with the aid of SPSS11.5 software package. A $P$ value of less than 0.05 was considered statistically significant.

\section{Results \\ Effect on immune function of Pi-deficiency mice}

The results showed that crude and processed licorice obviously improved the immune function of Pi-deficiency mice, including elevating expurgation index, spleen index and thymus index. The crude licorice improved the expurgation index was better than processed licorice, and the difference of expurgation index between crude and processed licorice group at the dosage of $5.0 \mathrm{~g} / \mathrm{kg}$ was significant $(\mathrm{P}<0.05)$, indicating that honey-roasting could enhance the licorice immunomodulation action. Crude licorice and processed licorice both increased the spleen and thymus index of Pi-deficiency mice, but the significant difference between the same dosage was not observed $(\mathrm{P}>0.05)$. While in the honey-treated groups, only the high dosage group was effective in improving the expurgation index of Pi-deficiency mice (Table 1).

Table 1: Effect of crude licorice, processed licorice and honey on the immune function of Pi-deficiency mice (mean $\pm \mathrm{SD}$, $n=10)$

\begin{tabular}{|c|c|c|c|c|}
\hline Group & Dose $(\mathrm{g} / \mathrm{kg})$ & $\begin{array}{ll}\text { Spleen index }(\mathrm{mg} / \mathrm{g}) \\
\end{array}$ & Thymus index (mg/g) & Expurgation index \\
\hline model & / & $3.24 \pm 0.54$ & $2.29 \pm 0.32$ & $5.31 \pm 2.28$ \\
\hline normal & l & $3.71 \pm 0.40^{*}$ & 2. $47 \pm 0.54$ & $7.59 \pm 2.40^{*}$ \\
\hline \multirow[t]{3}{*}{ Crude licorice } & 1.25 & $3.01 \pm 0.23$ & $2.13 \pm 0.49$ & $6.04 \pm 1.75$ \\
\hline & 2.50 & $3.84 \pm 0.67 *$ & $2.55 \pm 0.57$ & $7.61 \pm 2.73$ \\
\hline & 5.00 & $4.07 \pm 0.55 * *$ & $2.68 \pm 0.37 *$ & $8.19 \pm 2.53^{*}$ \\
\hline \multirow[t]{3}{*}{ Processed licorice } & 1.25 & $3.22 \pm 0.38$ & $2.28 \pm 0.35$ & $7.02 \pm 2.95$ \\
\hline & 2.50 & $4.03 \pm 0.66^{* *}$ & $2.48 \pm 0.46$ & $8.73 \pm 3.02^{*}$ \\
\hline & 5.00 & $4.26 \pm 0.73 * *$ & $2.77 \pm 0.41 * *$ & $10.43 \pm 2.91^{* * \#}$ \\
\hline \multirow{3}{*}{ Honey } & 1.25 & $3.42 \pm 0.44$ & $2.30 \pm 0.32$ & $5.25 \pm 1.62$ \\
\hline & 2.50 & $3.37 \pm 0.39$ & $2.44 \pm 0.46$ & $6.64 \pm 1.95$ \\
\hline & 5.00 & $3.54 \pm 0.45$ & $2.39 \pm 0.21$ & $7.56 \pm 1.20^{*}$ \\
\hline
\end{tabular}

* $\mathrm{P}<0.05,{ }^{* *} \mathrm{P}<0.01$ vs model group; ${ }^{\#} \mathrm{P}<0.05$ vs crude licorice group of the same dosage.

\section{Antitussive tests}

The results in Table 2 showed that crude and processed licorice obviously prolonged the cough incubation period and inhibited the cough frequency caused by ammonia, indicating that they had the significant antitussive activity. The action 
of crude licorice was obviously stronger than that of the same dosage processed licorice $(\mathrm{p}<0.05)$, implying that honey-roasting obviously weakened the licorice antitussive activity. In the honey-treated groups, the significant activity of honey was not observed.

Table 2: Effect of crude licorice, processed licorice and honey on mice cough induced by ammonia (mean $\pm \mathrm{SD}, n=10$ )

\begin{tabular}{llll}
\hline Group & Dose $(\mathrm{g} / \mathrm{kg})$ & Cough incubation period $(\mathrm{s})$ & Cough frequency (time) \\
\hline \multirow{2}{*}{ water } & $/$ & $35.9 \pm 11.8$ & $99.4 \pm 12.7$ \\
Crude licorice & 1.25 & $66.7 \pm 29.2^{*}$ & $61.2 \pm 20.3^{* *}$ \\
& 2.50 & $72.6 \pm 20.7^{* *}$ & $51.1 \pm 15.1^{* *}$ \\
Processed licorice & 5.00 & $76.4 \pm 22.8^{* *}$ & $48.4 \pm 10.0^{* *}$ \\
& 1.25 & $42.7 \pm 8.6^{\#}$ & $79.7 \pm 28.5$ \\
Honey & 2.50 & $47.2 \pm 11.7^{* \# \#}$ & $68.3 \pm 20.4^{* * \#}$ \\
& 5.00 & $55.8 \pm 21.3^{* \#}$ & $59.7 \pm 16.7^{* *}$ \\
& 1.25 & $36.9 \pm 15.4$ & $94.3 \pm 19.5$ \\
& 2.50 & $43.0 \pm 7.7$ & $83.5 \pm 21.6$ \\
& 5.00 & $40.9 \pm 13.8$ & $85.4 \pm 13.9$
\end{tabular}

*P $<0.05, * * \mathrm{P}<0.01$ vs vehicle group; ${ }^{\#} \mathrm{P}<0.05,{ }^{\#} \mathrm{P}<0.01$ vs crude licorice group of the same dosage.

Table 3: Effect of crude licorice, processed licorice and honey on the death caused by chloral hydrate

\begin{tabular}{ccc}
\hline Group & Dose $(\mathrm{g} / \mathrm{kg})$ & Survival rate \\
\hline water & $/$ & $4 / 20$ \\
Crude licorice & 7.50 & $10 / 20^{*}$ \\
Processed licorice & 7.50 & $8 / 20$ \\
Honey & 7.50 & $5 / 20$ \\
\hline
\end{tabular}

$* \mathrm{P}<0.05, * * \mathrm{P}<0.01$ vs vehicle group.

\section{Eliminating phlegm}

The results showed that the crude and processed licorice significantly enhanced the red phenol secretion of mice trachea, indicating they had the obvious activity of eliminating phlegm. The action intensity of crude licorice was weakened after processing. Honey didn’t show obvious effect (Figure 2).

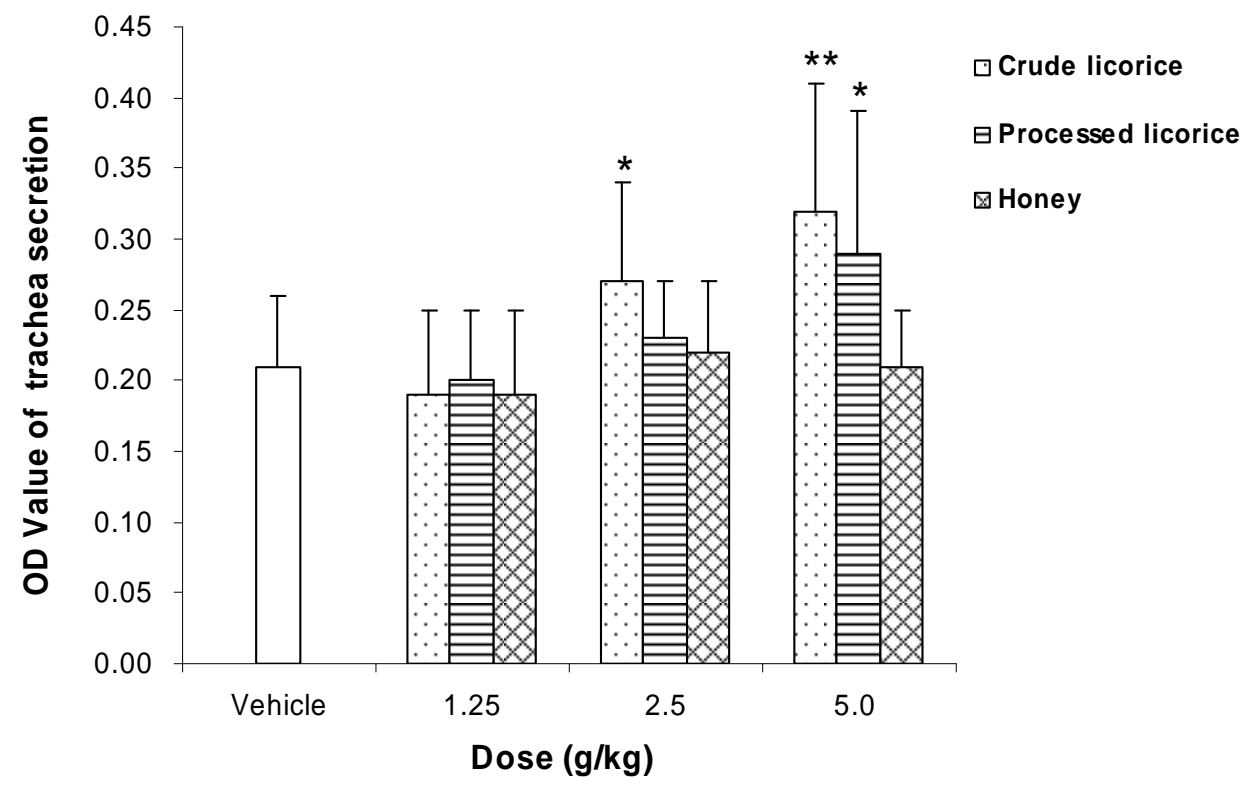

Figure 2: Effect of crude licorice, processed licorice and honey on red phenol secretion of mice trachea (mean $\pm \mathrm{SD}, n=10)$. $* \mathrm{P}<0.05, * * \mathrm{P}<0.01$ vs vehicle group.

\section{Detoxication test}

The results showed that crude licorice had the obvious detoxication action, it could inhibit the mice death caused by chloral hydrate. The obvious detoxication activity of processed licorice and honey was not observed (Table 3). The honey-roasting weakened licorice detoxication activity. 


\section{HPLC analysis}

The HPLC profiles (showed in Figure 3) showed the chemical constituents between crude and processed licorice,
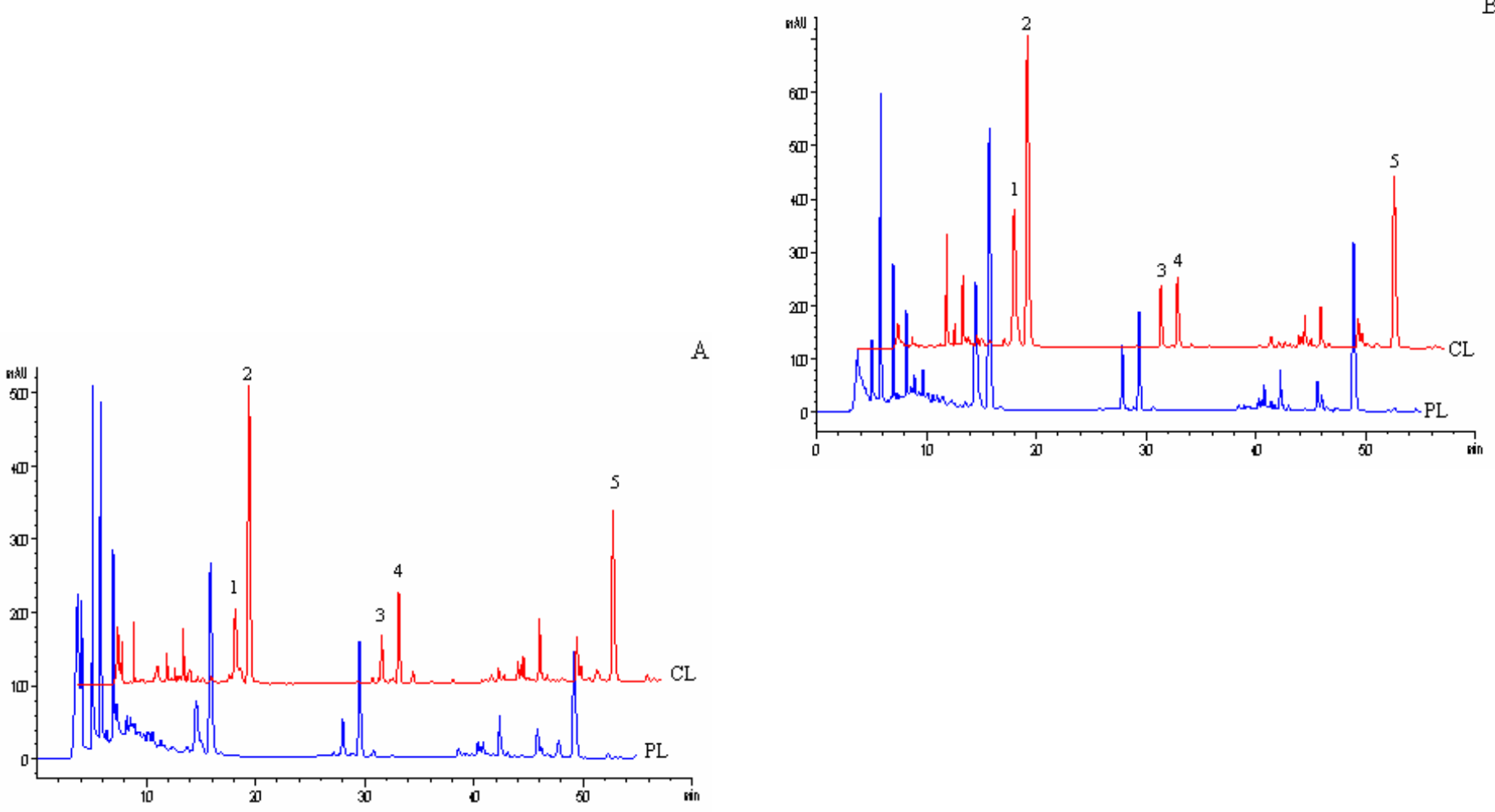

Figure 3: HPLC profiles of licorice (A. methanol extract of licorice; B. decoction of licorice; CL. crude licorice; PL. processed licorice; 1 . liquiritin apioside; 2. liquiritin; 3. licuraside; 4. isoliquiritin; 5. glycyrrhizin). The mobile phase consisted of acetonitrile (a) and $0.1 \%$ phosphoric acid solution $(\mathrm{v} / \mathrm{v}, \mathrm{b})$. The gradient and detection wavelength were as follows: 0-20 min, 13\%-19\% a, $276 \mathrm{~nm}$; 20-37 min, $19 \%-35 \%$ a, $360 \mathrm{~nm}$; $37-55 \mathrm{~min}, 35 \%-55 \%$ a, $254 \mathrm{~nm}$; flow rate: $0.8 \mathrm{ml} / \mathrm{min}$. The injection volume was $10 \mu \mathrm{L}$ and the column was thermostated at 35 .
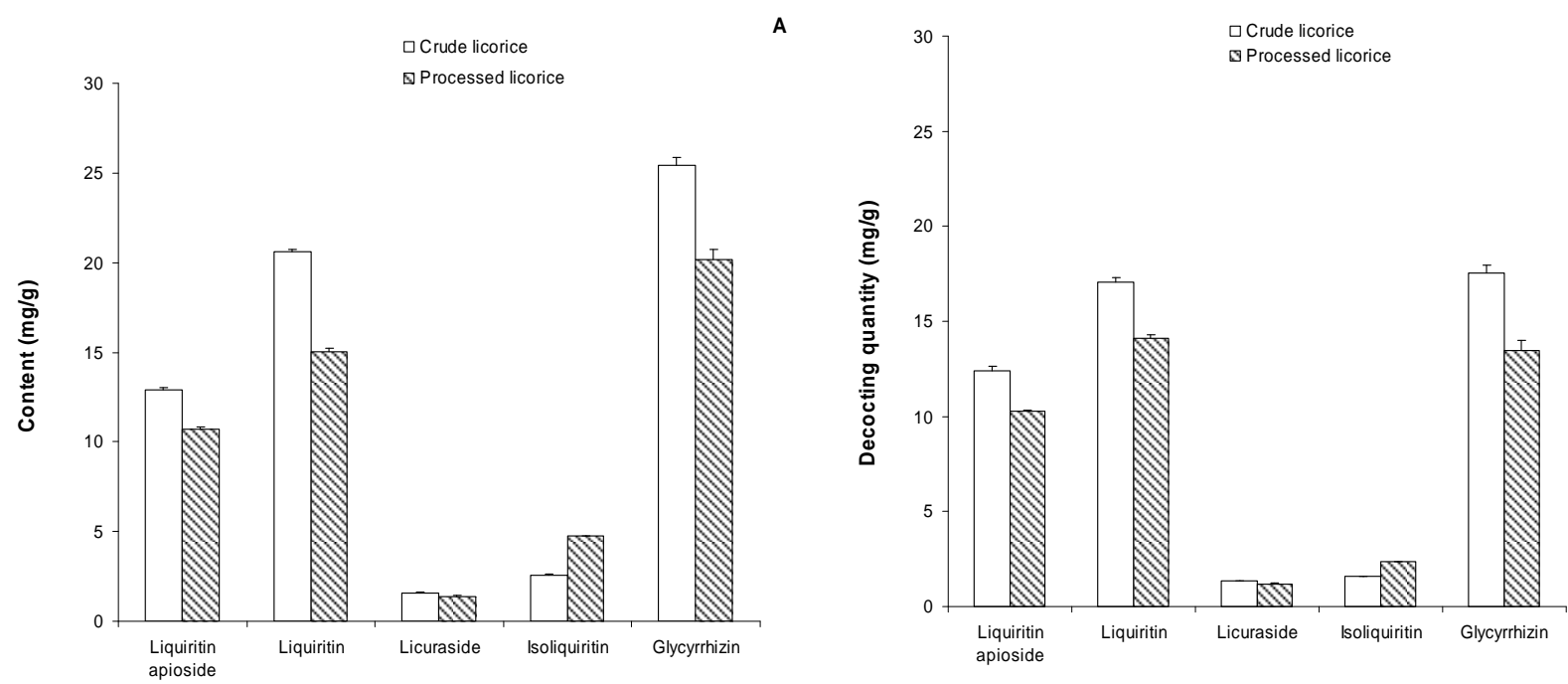

Figure 4: Influence of processing on the content (A) and decocting quantity (B) of five active compounds in licorice (mean \pm 
$\mathrm{SD}, n=3)$. Content $=$ the compound weight $(\mathrm{mg})$ detected in licorice/ licorice weight $(\mathrm{g})$; Decocting quantity $=$ the compound weight $(\mathrm{mg})$ detected in water decoction/ licorice weight $(\mathrm{g})$.

were very different. The polar constituents of processed licorice and its decoction were more abundant than those of crude licorice. The ratio of peak areas, such as licuraside (3)/isoliquiritin (4), obviously altered after processing. The result indicated that honey-roasting modified the chemical compositions in licorice. The contents of five compounds in licorice slice and the decocting quantity were obtained, according to the standard calibration curves established from six different concentrations of the standard solutions. The results showed that the contents and decocting quantity of liquiritin apioside, liquiritin and glycyrrhizin in processed licorice descended significantly, while the content and decoction of isoliquiritin obviously increased (Figure 4). In order to determine if honey-roasting had caused the decomposition of active glycosides, the standards were treated under the same processing condition. HPLC analysis results (Figure 5) showed that liquiritin and glycyrrhizin were obviously decomposed. Glycyrrhizin was decomposed to glycyrrhetic acid-3-O- $\beta$-D-glucuronide and glycyrrhetic acid. Liquiritin produced isoliquirtin and liquiritigenin. The result indicated that honey-roasting altered the chemical structure of active glycosides in licorice.

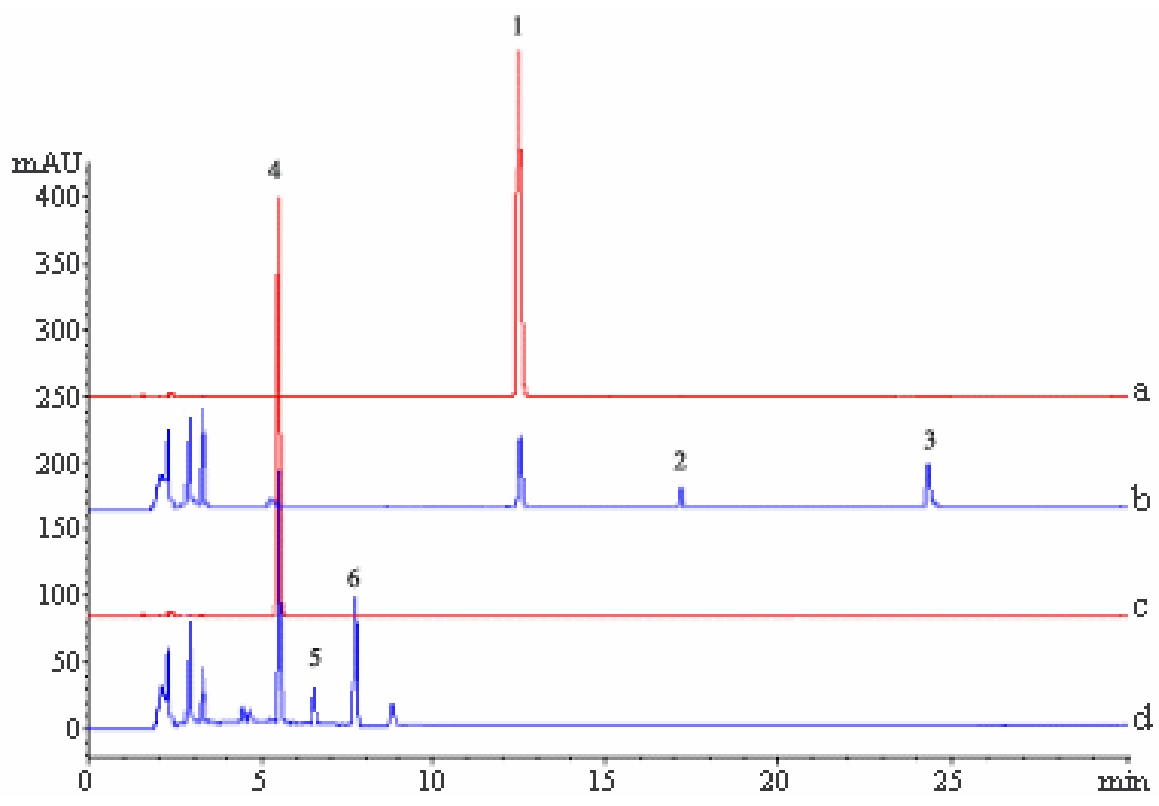

Figure 5: HPLC profiles of liquiritin and glycyrrhizin before and after honey-roasting (a. glycyrrhizin + honey, under room temperature for $1 \mathrm{~h}$; b. glycyrrhizin + honey, under 180 for $1 \mathrm{~h}$; c. liquiritin + honey, under room temperature for $1 \mathrm{~h}$; d. liquiritin + honey, under 180 for $1 \mathrm{~h} ; 1$. glycyrrhizin; 2. glycyrrhetic acid-3-O-glucuronide; 3. glycyrrhetic acid; 4. liquiritin; 5. isoliquiritin; 6 . liquiritigenin. The mobile phase consisted of acetonitrile (a) and $0.1 \%$ aqueous methanoic acid (v/v, b). The gradient was as follows: 0-30 min, 20\%-100\% a, $1.0 \mathrm{ml} / \mathrm{min}$. Detection wavelength was $254 \mathrm{~nm}$ and the column was thermostated at 35 . The injection volume was $5 \mu$ l.

\section{Discussion}

Licorice is one of the most common medicines prescribed by TCM practitioners. TCM theory claims that its efficacy obviously changes after processing with honey. Since $200 \mathrm{BC}$, crude licorice and processed licorice have been used separately, and have been two of the most frequently employed botanicals in China (China Pharmacopoeia Committee, 2005). According to TCM theory, the crude licorice has the stronger potency of relieving cough, eliminating phlegm and detoxication. The complex prescriptions for cough and cold, such as Sangju drink, Yinqiao powder and Chaige Jieji decoction, often use crude licorice. Nowadays, the crude licorice has been used widely for bronchitis and asthma in Eastern and Western cultures. Our study showed that the honey-roasting weakened the crude licorice potency in relieving cough, eliminating phlegm and detoxication for the first time. The result was consisted with licorice clinical use.

It is reported that glycyrrhizin, liquiritin apioside and liquiritin have the significant activity of relieving cough and inhibiting allergic asthma (Kamei et al., 2005; Jayaprakasam et al., 2009; Xie et al., 2009; Shin et al., 2007). Their content decreases significantly after roasting with honey (Sung and Li, 2004; Wang et al., 1995; Wang et al., 2008). This study revealed that the activity of relieving cough and eliminating phlegm weakened obviously after honey processing; the contents and decocting quality of liquiritin apioside, liquiritin and glycyrrhizin descended obviously; honey did not show obvious activity. The activity decrease after honey processing was probably associated with the content decrease of flavonoid 
glycosides and glycyrrhizin.

In TCM theory, crude licorice is good at detoxication also. This study showed the crude product had the stronger activity of inhibiting death rate caused by chloral hydrate than that of processed product. It is reported that glycyrrhizin cloud relief acute intoxication caused by food and drug, and its content in crude licorice is higher than that in processed licorice (Li and Tong, 2007; He et al., 2009; Rahman and Sultana, 2007). Our study showed that the content and decocting quantity of glycyrrhizin in licorice descended after processing, and honey did not possess obvious intoxication action. So, the difference in detoxication activity between crude and processed licorice may be associated with the content of glycyrrhizin.

Processed licorice is believed to possess the better effect of invigorating vital energy than crude licorice, and often used to cure Pi-deficiency syndrome in TCM clinic. Some complex prescriptions for Pi-deficiency syndrome, such as Buzhong Yiqi decoction and Sijunzi decoction, all require using processed licorice rather than crude licorice. Although crude licorice and processed licorice had the obvious effect of enhancing Pi-deficiency mice's immune function, the processed product showed the stronger activity than crude licorice in the present study. Glycyrrhizin and polysaccharides have been reported to possess significant activity of enhancing immune function (Yi et al., 1996; Cheng et al., 2008), but their content decrease after roasting with honey. It is to be noted that the content and decocting quantity of isoliquiritin increased obviously after processing in the present study. So, the immunomodulation activity of isoliquiritin is worth investigating.

The results of content analysis in the present study indicated that glycyrrhizin and flavone glycosides may be decomposed or isomerized in the processing condition. In order to test this hypothesis, the stability of glycyrrhizin and liquiritin, two major ingredients in licorice, was investigated under the same processing condition. The results indicated that the honey-roasting did cause the decompostion and isomerization of the active glycosides in licorice. The recently studies showed that roasting could caused glycyrrhizin thermal decomposition (Sung and Li, 2004; Hwang et al., 2006), consisted with the result of the present study. Previous research indicated that glycosidic flavonoid constituents were hydrolyzed stepwise during roasting (Kuwajima, 1999). Our study found that liquiritin isomerized to isoliquiritin and decomposed to liquiritigenin during honey-roasting for the first time. This conversion reaction was consisted with the content increase of isoliquiritin in processed licorice.

In conclusion, the honey-roasting obviously modified the main pharmacological activities and chemical compositions of licorice. This modification didn't cause by honey only, and the roasting appeared to exert some effects. These findings provided a piece of scientific proof for the different therapeutic values of crude and processed licorice. In order to elucidate the mechanism of roasting with honey, the more researches, including the investigation of the processing influence on the lipid- soluble active ingredients, the absorption and metabolism of the active compounds in licorice, should be carried out.

\section{Acknowledgment}

This work was supported by the National Natural Science Foundation of China (No. 81173551; No. 30973962).

\section{References}

1. Chen, Q. (1994). Pharmacological experiment on Chinese traditional medicine. Beijing: People's Medical Publishing House, 56-57.

2. Cheng, A.W., Wan, F.C., Wang, J.Q., Jin, Z.Y. and Xu, X.M. (2008). Macrophage immunomodulatory activity of polysaccharides isolated from Glycyrrhiza uralensis Fish. Int. Immunopharmacol., 8 (1): 43-50.

3. China Pharmacopoeia Committee (2005). Chinese Pharmacopoeia (Vol ). Beijing: Chemical Industry Press, 59-60.

4. Gong, Q.F. (2007). Processing of traditional Chinese medicine. Beijing: China Press of Traditional Chinese Medicine, 220-222.

5. He, D., Liu, F.Q. and Li, H.D. (2009). Progress of study on licorice detoxication action. Cent. South Pharm., 7 (12): 927-931.

6. Huang, W.L., Tan, S.D., Li, W.H., Zhu, X.F., Hu, C.J., and Cao, G.B. (1984). The effect of crude and processed licorice on experimental arhythmia. Chin. Trad. Pat. Med., 6 (6): 13-15.

7. Hwang, I.K., Lim, S.S., Choi, K.H., Yoo, K.Y., Shin, H.K., Kim, E.J., Park, J.H.Y., Kang, T.C., Kim, Y.S., Kwon, D.Y., Kim, D.W., Moon, W.K. and Won, M.H. (2006). Neuroprotective effects of roasted licorice, not raw form, on neuronal injury in gerbil hippocampus after transient forebrain ischemia. Acta Pharmacol. Sin., 27 (8): 959-965.

8. Jayaprakasam, B., Doddaga, S., Wang, R., Holmes, D., Goldfarb, J. and Li, X.M. (2009). Licorice flavonoids inhibit Eotaxin-1 secretion by human fetal lung fibroblasts in vitro. J. Agric. Food Chem., 57 (3): 820-825.

9. Kamei, J., Saitoh, A., Asano, T., Nakamura, R., Ichiki, H., Iiduka, A. and Masayoshi, K. (2005). Pharmacokinetic and pharmacodynamic profiles of the antitussive principles of Glycyrrhizae radix (licorice), a main component of the Kampo preparation Bakumondo-to (Mai-men-dong-tang). Eur. J. Pharmacol., 507(1-3): 163-168.

10. Kim, J.K., Oh, S.M., Kwon, H.S., Oh, Y.S., Lim, S.S. and Shin, H.K. (2006). Anti-inflammatory effect of roasted licorice extracts on lipopolysaccharide-induced inflammatory responses in murine macrophages. Biochem. Biophys. Res. Commun., 345 (3): 1215-1223.

11. Kim, K.R., Jeong, C.K., Park, K.K., Choi, J.H., Park, J.H., Lim, S.S. and Chung, W.Y. (2010). Anti-inflammatory effects of licorice and roasted licorice extracts on TPA-induced acute inflammation and collagen-induced arthritis in mice. J. Biomed. Biotech., 2010, Article 709378.

12. Ko, B.S., Jang, J.S., Hong, S.M., Sung, S.R., Lee, J.E., Lee, M.Y., Jeon, W.K. and Park S. (2007). Changes in components, glycyrrhizin and glycyrrhetinic acid, in raw Glycyrrhiza uralensis Fisch, modify insulin sensitizing and insulinotropic actions. Biosci. Biotech. Bioch., 71 (6): 1452-1461. 
13. Kuwajima, H., Taneda, Y., Chen, W.Z., Kawanishi, T., Hori, K., Taniyama, T., Kobayashi, M., Ren, J. and Kitagawa, I. (1999). Variation of chemical constituents in processed licorice roots: Quantitative determination of saponin and flavonoid constituents in bark removed and roasted licorice roots. Yakugaku Zasshi, 119 (12): 945-955.

14. Li, B.X. and Tong, J. (2007). Progress of study on pharmacological activities and mechanisms of glycyrrhizic acid. J. Math. Med., 20 (2): 228-231.

15. Liu, Y.Q., Wang M.Y., Shi H.M., Sun Q.S., and Li X.B. (2008). Determination of five active constituents in licorice and its processed products. Chin. Pharm. J., 43 (16): 1268-1271.

16. Majima, T., Yamada, T., Tega, E., Sakurai, H., Saiki, I. and Tadato, T. (2004). Pharmaceutical evaluation of liquorice before and after roasting in mice. J. Pharm. Pharmacol., 56 (5): 589-595.

17. Peng, Z.C., Lu, H.L. and Yi, S.F. (1989). The analgesic effect of processed licorice. Chin. J. Chin. Mater. Med., 14 (8): 22-23.

18. Rahman, S. and Sultana, S. (2007). Glycyrrhizin exhibits potential chemopreventive activity on 12-O-tetradecanoyl phorbol-13-acetate-induced cutaneous oxidative stress and tumor promotion in swiss albino mice. J. Enzym. Inhib. Med. Chem., 22 (3): 363-369.

19. Sanders, V.M., Kauffmann, B.M., White, K.L., Douglas, J.K., Barnes, D.W., Sain, L.E., Bradshaw, T.J., Borzelleca, J.F. and Munson, A. E. (1982). Toxicology of chloral hydrate in the mouse. Environ. Health Perspect., 44 (4): 137-146.

20. Shang, H.P., Cao, S.H., Wang, J.H., Zheng, H. and Putheti, R. (2010). Glabridin from Chinese herb licorice inhibits fatigue in mice. Afr. J. Trad. CAM, 7 (1): $17-23$.

21. Shin, Y.W., Bae, E.A., Lee, B., Lee, S.H., Kim, J.A., Kim, Y.S. and Kim, D.H. (2007). In vitro and in vivo antiallergic effects of Glycyrrhiza glabra and its components. Planta Med., 73 (3): 257-261.

22. Sung, M.W. and Li, P.C. (2004). Chemical analysis of raw, dry-roasted, and honey-roasted licorice by capillary electrophoresis. Electrophoresis, 25 (20): 3434-3440.

23. Wang, J.Z., Chen, D.Y. and Zhao, X.H. (1995). Analytical study on drug-processing of the root of Glycyrrhiza uralensis Fisch by HPLC. Chin. J. Chin. Mater. Med., 20 (9): 535-537.

24. Wang, M.Y., Liu, Y.Q., Shi, H.M. and Li, X.B. (2008). Determination of five active constituents in licorice and its processed products. Chin. Pharm. J., 43 (16): 1268-1271.

25. Xie, Y.C., Dong, X.W., Wu, X.M., Yan, X.F. and Xie, Q.M. (2009). Inhibitory effects of flavonoids extracted from licorice on lipopolysaccharide-induced acute pulmonary inflammation in mice. Int. Immunopharmacol., 9 (2): 194-200.

26. Xu, S.Y., Bian, R.L. and Chen, X. (2002). Pharmacological Experiment Methodology. Beijing: People's Medical Publishing House, 1359-1364.

27. Yi, H., Nakashima, I. and Isobe, K. (1996). Enhancement of nitric oxide production from activated macrophages by glycyrrhizin. Am. J. Chin. Med., 24 (3/4): 271-278.

28. Zheng, X.W., Wang, Y. and Song, H. (2006). Experimental study on effect of Buzhong Yiqi decoction on serum gastrin in spleen-qi deficiency rats. Chin. J. Trad. Chin. Med. Pharm., 21 (7): 393-395. 